\title{
Propagation properties of inertia-gravity waves through a barotropic shear layer and application to the Antarctic polar vortex
}

\author{
By M. C. ÖLLERS ${ }^{1,3}$, L. P. J. KAMP ${ }^{*}$, F. LOTT ${ }^{2}$, P. F. J. VAN VELTHOVEN ${ }^{3}$, H. M. KELDER ${ }^{1,3}$ and \\ F. W. SLUIJTER ${ }^{1}$ \\ ${ }^{1}$ Department of Applied Physics, Eindhoven University of Technology, the Netherlands \\ ${ }^{2}$ Laboratoire de Météorologie Dynamique du CNRS, Université Pierre et Marie Curie, Paris, France \\ ${ }^{3}$ Royal Netherlands Meteorological Institute, De Bilt, the Netherlands
}

(Received 3 April 2002; revised 19 December 2002)

SUMMARY

The propagation of inertia-gravity waves (IGWs) through a dynamical transport barrier, such as the Antarctic polar vortex edge is investigated using a linear wave model. The model is based on the linearized, inviscid hydrostatic equations on an $f$-plane. Typical values for the parameters that are appropriate to the Antarctic polar vortex are given. The background flow $U$ is assumed to be barotropic and its horizontal shear is represented by a hyperbolic tangent background wind profile. The wave equation that describes the latitudinal structure of a monochromatic disturbance contains two singularities. The first corresponds to the occurrence of a critical level where the intrinsic wave frequency $\Omega=\omega-k U$ becomes zero. $\omega$ is the absolute wave frequency and $k$ its longitudinal wave number in the direction of $U$. The second is an apparent singularity and does not give rise to singular wave behaviour. It becomes zero whenever the square of the intrinsic wave frequency $\Omega^{2}=f\left(f-U_{y}\right)$, $f$ being the Coriolis frequency and $U_{y}$ the horizontal shear of the flow. The wave equation is solved numerically for different values of the angles of incidence of the wave upon the background flow, of the wave frequency, of the horizontal wave number and of the Rossby number. Reflection $(|R|)$ and transmission $(|T|)$ coefficients are determined as a function of these parameters. The results depend on whether the flow is inertially stable or not. They also depend on the presence and location of the turning levels, where the wave becomes evanescent, with respect to the location of the $\Omega$-critical levels. For inertially stable flows, the wave totally reflects at the turning level and never reaches the critical level. If the background flow is inertially unstable, turning levels can disappear and the wave can now reach the critical level. Then over-reflection, over-transmission and absorption can occur.

KEYWORDS: Critical layers Over-reflection Over-transmission Resonant absorption

\section{INTRODUCTION}

The dynamics of the Antarctic polar vortex in the lower and middle stratosphere have been much studied during the last 20 years (e.g. Schoeberl and Hartmann 1991; Randel 1993; McIntyre 1995). They follow early studies by, for example, Juckes and McIntyre (1987) and McIntyre (1989) which have shown that such a vortex behaves like a dynamical barrier for atmospheric minor constituents.

The Antarctic polar vortex is characterized by a strong gradient in potential vorticity (the vortex edge) which is nearly impermeable to transports induced by large-scale motions (e.g. Bowman 1993; Chen 1994; Öllers et al. 2002), but can be more porous to transports related to smaller-scale inertia-gravity waves (IGWs) (McIntyre 1995). Nevertheless, very few studies have actually addressed how IGWs dynamically interact with such a vortex. Dunkerton (1984) investigated the propagation and refraction properties of stationary IGWs in zonal-mean flows in the stratosphere using a ray-tracing technique. Pierce et al. (1994) performed Lagrangian material line calculations and investigated the impact of an idealized IGW field on irreversible mixing and stretching of material lines near the Arctic and Antarctic vortex edges. More fundamentally but still in the context of wave-barrier interaction, Staquet and Huerre (2001) analysed the breaking of IGWs in a rotating barotropic flow with horizontal shear near an $N$-critical level, where the intrinsic wave frequency $(\Omega)$ equals the Brunt-Väisälä

* Corresponding author: Department of Applied Physics, Eindhoven University of Technology, PO Box 513, NL-5600 MB, Eindhoven, the Netherlands. e-mail: L.P.J.Kamp@tue.nl

(c) Royal Meteorological Society, 2003. 
frequency $(N)$, by using a three-dimensional Boussinesq fully nonlinear model. The interaction between oceanic internal gravity waves and barotropic background flows with horizontal shear has been studied by Ivanov and Morozov (1974), Olbers (1981) and Basovich and Tsimring (1984). Basovich and Tsimring (1984) showed, using the linearized Boussinesq equations, that, depending on specific wave and background flow characteristics, a wave may be partially absorbed (wave energy is released into the background flow), totally reflected (no energy exchange) or over-reflected (the wave extracts energy from the background flow) at $N$-critical levels $(\Omega=N)$ or $\Omega$-critical levels $(\Omega=0)$. These types of critical levels have not been studied much in the past for IGWs propagating towards a barotropic flow. A plausible reason for this is that in most circumstances the critical levels are preceded by turning levels where Wentzel, Kramers, Brillouin (WKB) theory predicts total reflection. Depending on whether the Coriolis frequency $f$ is assumed to be constant or changing with latitude, waves can, respectively, be absorbed (Jones 1967) or reflected (e.g. Kitchen and McIntyre 1980) at Jones critical levels $(\Omega=f)$.

Although the interaction of IGWs with a barotropic jet is little studied, the propagation of internal gravity waves in background flows with vertical shear has been extensively studied since the seminal paper of Booker and Bretherton (1967). In the presence of an $\Omega$-critical level the value of the Richardson number plays a key role in the stability of the flow and determines whether waves will be absorbed (Booker and Bretherton 1967) or over-reflected at the critical level (Jones 1968; Acheson 1976; van Duin and Kelder 1982).

In the present study, the interaction between monochromatic IGWs and a barotropic background flow is studied in a linear hydrostatic model. The background flow is represented by a hyperbolic tangent profile. The same qualitative behaviour, with respect to the reflection and transmission properties, is found for jet-type profiles mimicking, for example, the Antarctic polar vortex edge.

From the basic model equations a wave equation is derived that describes this interaction. The wave equation that describes the disturbance field is the $f$-plane version of Laplace's tidal equation with shear in the zonal background flow in the latitudinal direction (Flattery 1967; Longuet-Higgins 1968). It contains two singularities that are of different natures. One is similar to that found by Booker and Bretherton (1967). The second one is decribed by Boyd (1978) and Dunkerton (1990) for the case when the background flow has merely latitudinal shear, by Yamanaka and Tanaka (1984) for the case of a vertically sheared background flow, and the combined case of latitudinally and vertically sheared background flows is described by Kitchen and McIntyre (1980). This singularity, denoted as $A$ here or $-\Delta$ in Boyd (1978) and Dunkerton (1990), occurs whenever the square of the intrinsic wave frequency $\Omega$ equals $f(f-\mathrm{d} U / \mathrm{d} y), \mathrm{d} U / \mathrm{d} y$ is the shear of the background flow $U$ in the latitudinal direction. It has been shown by Boyd (1976) that the zeroes of $A$ are apparent singularities. No jump in the momentum flux is associated with these apparent singularities.

The parameters are chosen to compare with the Antarctic polar vortex. Reflection and transmission coefficients are determined for waves incident upon the background flow as a function of several characteristic parameters, and are discussed with regard to the degree of inertial instability, which is measured here by a Rossby number.

Although our model is used to study the basic interactions between IGWs and the Antarctic polar vortex, its range of application is much wider and may also be used to study the interaction between IGWs and horizontally sheared background flows on an $f$-plane in more general conditions such as at midlatitudes and the tropics (e.g. Dunkerton 1990 and references therein). 
This paper should be considered as a first approach to understanding the basic mechanisms of the interaction between IGWs and strong barriers like the Antarctic polar vortex edge and their effect upon its permeability.

The paper is organized as follows. In section 2 the mathematical background of our model is presented. In section 3 some details are given on the numerical integration procedure. The choice of typical values for the parameters are outlined and the main numerical results are at the end of section 3. In the final section we summarize our major results.

\section{MODEL FORMULATION}

(a) Basic model equations

The linearized equations in log-pressure coordinates for hydrostatic perturbations in a horizontal shear flow $U(y)$ are,

$$
\begin{gathered}
\frac{\partial u}{\partial t}+U \frac{\partial u}{\partial x}+v \frac{\mathrm{d} U}{\mathrm{~d} y}-f v=-\frac{\partial \phi}{\partial x}, \\
\frac{\partial v}{\partial t}+U \frac{\partial v}{\partial x}+f u=-\frac{\partial \phi}{\partial y}, \\
\frac{\partial^{2} \phi}{\partial t \partial z}+U \frac{\partial^{2} \phi}{\partial x \partial z}+N^{2} w=0 \\
\frac{\partial u}{\partial x}+\frac{\partial v}{\partial y}+\frac{\partial w}{\partial z}-\frac{w}{H}=0,
\end{gathered}
$$

where $u, v$ and $w$ are latitudinal, longitudinal and vertical velocity perturbations, respectively, $\phi$ corresponds to the geopotential, $f$ is the Coriolis frequency and $H$ is the vertical scale height. In Eq. (3) $N^{2}=\left(g / \theta_{0}\right) \mathrm{d} \theta_{0} / \mathrm{d} z$ is the Brunt-Väisälä frequency, where $g$ is the gravitational acceleration and $\theta_{0}$ is the background potential temperature. Hereafter, it is assumed that $N^{2}$ is constant, which is a reasonable approximation since in the lower and middle stratosphere $\theta_{0}$ is a nearly linear function of height (Andrews et al. 1987). The assumption of hydrostatic equilibrium is justified here since we are primarily interested in low-frequency IGWs with horizontal scales much larger than the vertical scales. Thus, the possibility that $N$-critical levels occur is excluded.

Finally, $U(y)$ is the background flow, which is assumed to be independent of $z$ and to have a barotropic shear in the horizontal $y$-direction only. In the following, $U(y)$ will be modelled by a hyperbolic tangent or transitional profile,

$$
U(y)=\frac{U_{0}}{2}\{1+\tanh (y / 2 L)\} .
$$

In Eq. (5), $U_{0}$ is a characteristic maximum velocity of the background flow and $L$ is a measure of the shear layer width.

Assume now wave-like disturbances $\zeta(x, y, z, t)=\widehat{\zeta}(y) \mathrm{e}^{\{\mathrm{i}(\omega t-k x-m z)+z / 2 H\}}$, where $\zeta$ is a general notation for $u, v, w$ and $\phi$. After substitution in Eqs. (1) through (4), the following wave equation for $\widehat{\phi}$ can be derived

$$
\frac{\mathrm{d}^{2} \widehat{\phi}}{\mathrm{d} y^{2}}-\frac{1}{A} \frac{\mathrm{d} A}{\mathrm{~d} y} \frac{\mathrm{d} \widehat{\phi}}{\mathrm{d} y}+\left(\frac{A}{\Lambda^{2}}-\frac{k f}{A \Omega} \frac{\mathrm{d} A}{\mathrm{~d} y}-k^{2}\right) \widehat{\phi}=0,
$$

with

$$
\Omega=(\omega-k U), \quad A=\Omega^{2}-\omega_{i}^{2}, \quad \Lambda^{2}=N^{2} /\left(1 / 4 H^{2}+m^{2}\right) .
$$


In Eq. (7) $\omega_{i}^{2}$ is defined as

$$
\omega_{i}^{2}=f(f-\mathrm{d} U / \mathrm{d} y) .
$$

Equation (6) becomes singular whenever the intrinsic wave frequency $\Omega$ is equal to zero. In that case the horizontal phase speed of the wave equals that of the background flow. Note that this type of singularity is similar to the classical Booker and Bretherton type singularity for internal gravity waves propagating in a horizontally uniform wind field with vertical shear (Booker and Bretherton 1967). However, the $\Omega$-singularity in our case is a logarithmic singularity (see appendix), whereas the Booker and Bretherton type singularity is non-logarithmic. Note that $A=0$ will not give rise to singular behaviour in $\widehat{\phi}$, since $A$ is an apparent singularity. We note that the background flow becomes inertially unstable if $\omega_{i}^{2}<0$ for certain values of $y$.

Equation (6) can be written into its normal form using $\widehat{\phi}(y)=A^{1 / 2} \widehat{\psi}(y)$, i.e.

$$
\frac{\mathrm{d}^{2} \psi}{\mathrm{d} y^{2}}+\underbrace{\left\{-\frac{3}{4 A^{2}}\left(\frac{\mathrm{d} A}{\mathrm{~d} y}\right)^{2}+\frac{1}{2 A} \frac{\mathrm{d}^{2} A}{\mathrm{~d} y^{2}}+\frac{A}{\Lambda^{2}}-\frac{k f}{A \Omega} \frac{\mathrm{d} A}{\mathrm{~d} y}-k^{2}\right\}}_{Q(y)} \psi=0 .
$$

Turning levels occur at locations where $Q(y)=0$. Note that $Q(y)$ will become singular if $\Omega=0$ and $A=0$.

\section{(b) Conservation of momentum fluxes}

Equation (6) can be rewritten in the form

$$
\frac{\mathrm{d}}{\mathrm{d} y}\left(\frac{1}{A} \frac{\mathrm{d} \widehat{\phi}}{\mathrm{d} y}\right)+\underbrace{\left\{\frac{1}{\Lambda^{2}}-\frac{k f}{A^{2} \Omega} \frac{\mathrm{d} A}{\mathrm{~d} y}-\frac{k^{2}}{A}\right\}}_{P} \widehat{\phi}=0 .
$$

Now, multiply Eq. (10) by i $\widehat{\phi}^{*}$ yielding

$$
\frac{\mathrm{d}}{\mathrm{d} y}\left(\frac{\mathrm{i} \widehat{\phi}^{*}}{A} \frac{\mathrm{d} \widehat{\phi}}{\mathrm{d} y}\right) \underbrace{-\frac{\mathrm{i}}{A} \frac{\mathrm{d} \widehat{\phi}^{*}}{\mathrm{~d} y} \frac{\mathrm{d} \widehat{\phi}}{\mathrm{d} y}+\mathrm{i} P \widehat{\phi} \widehat{\phi}^{*}}_{\text {purely imaginary }}=0,
$$

where the asterisks indicate complex conjugation. It now follows that

$$
C=\operatorname{Re}\left(\frac{\mathrm{i} \widehat{\phi}^{*}}{A} \frac{\mathrm{d} \widehat{\phi}}{\mathrm{d} y}\right),
$$

which does not depend on $y$ as long as $\Omega \neq 0$. Also, the quantity in Eq. (11) is closely related to a momentum flux. From Eqs. (1) and (2) it can be derived that the momentum flux

$$
\tau \equiv\left\langle\rho_{0} u v\right\rangle=\rho_{0} \operatorname{Re}\left(\frac{\mathrm{i} k \widehat{\phi}^{*}}{A} \frac{\mathrm{d} \widehat{\phi}}{\mathrm{d} y}\right),
$$

where $\rho_{0}$ corresponds to the background density of air and \langle\rangle denotes an average over a wave cycle.

An IGW incident upon the transitional wind profile (Eq. (5)) from $y \rightarrow-\infty$ gives rise to a reflected wave there and transmitted wave at $y \rightarrow \infty$. The solution for $y \rightarrow-\infty$ 
reads

$$
\widehat{\phi}(y)=\exp \left(-\mathrm{i} l_{-\infty} y\right)+R \exp \left(\mathrm{i} l_{-\infty} y\right)
$$

the amplitudes of the incident and reflected waves being 1 and $R$, respectively. $l_{-\infty}$ corresponds to the horizontal wave number in the $y$-direction evaluated at $y=-\infty$ and is defined by

$$
l_{-\infty}=\left(\frac{\left(\omega^{2}-f^{2}\right)}{\Lambda^{2}}-k^{2}\right)^{1 / 2}
$$

For $y \rightarrow \infty$ the solution takes the form

$$
\widehat{\phi}(y)=T \exp \left(-\mathrm{i} l_{+\infty} y\right),
$$

where $T$ is the amplitude of the transmitted wave and $l_{+\infty}$ is defined by

$$
l_{+\infty}=\left(\frac{\left\{\left(\omega-k U_{0}\right)^{2}-f^{2}\right\}}{\Lambda^{2}}-k^{2}\right)^{1 / 2},
$$

and means the value $l$ takes as $y \rightarrow \infty$. In case Eq. (6) has no real singularities $(\Omega \neq 0)$, we have

$$
C_{-\infty}=C_{+\infty}
$$

with

$$
C_{-\infty}=\left.\operatorname{Re}\left(\frac{\mathrm{i}}{A} \widehat{\phi}^{*} \frac{\mathrm{d} \widehat{\phi}}{\mathrm{d} y}\right)\right|_{y=-\infty}, \quad C_{+\infty}=\left.\operatorname{Re}\left(\frac{\mathrm{i}}{A} \widehat{\phi}^{*} \frac{\mathrm{d} \widehat{\phi}}{\mathrm{d} y}\right)\right|_{y=+\infty} .
$$

Using the results Eqs. (13) through (18) one derives for the hyperbolic tangent profile (Eq. (5)) that

$$
|R|^{2}+\frac{l_{+\infty}}{A_{+\infty}} \frac{A_{-\infty}}{l_{-\infty}}|T|^{2}=1 .
$$

For IGWs incident upon a symmetrical jet-type wind profile we would have that $A_{-\infty}=A_{+\infty}$ and $l_{-\infty}=l_{+\infty}$, resulting in

$$
|R|^{2}+|T|^{2}=1
$$

If $\Omega$ does become zero (i.e. $\Omega$-critical levels are present) for particular values of $y$ between $y=-\infty$ and $y=+\infty$, it is expected that the quantity $C$ in Eq. (11) is discontinuous at these locations (critical levels). In that case Eq. (19) should fulfil

$$
|R|^{2}+\frac{l_{+\infty}}{A_{-\infty}} \frac{A_{+\infty}}{l_{-\infty}}|T|^{2}=1+\frac{A_{-\infty}}{l_{-\infty}}\left(C_{+\infty}-C_{-\infty}\right) .
$$

Equation (21) shows that the sum of $|R|^{2}$ and $(l / A)_{\infty}(A / l)_{-\infty}|T|^{2}$ may differ from 1 depending on the magnitude of the jump $\left(A_{-\infty} / l_{-\infty}\right)\left(C_{+\infty}-C_{-\infty}\right)$ in passing critical levels. Whenever $\Omega$ becomes zero somewhere between $y=-\infty$ and $y=+\infty$, the momentum flux $\tau$ in Eq. (12) will jump in passing these $\Omega$-critical levels. Analytical expressions for such jumps are given in the appendix. There it is shown that the sign of $\left(C_{+\infty}-C_{-\infty}\right)$ depends upon the sign of $\left(\mathrm{d}^{2} U / \mathrm{d} y^{2}\right) /\left.(\mathrm{d} U / \mathrm{d} y)\right|_{y=y_{\mathrm{c}}}$, where $y_{\mathrm{c}}$ is the location of the $\Omega$-critical level. If $\left(\mathrm{d}^{2} U / \mathrm{d} y^{2}\right) /\left.(\mathrm{d} U / \mathrm{d} y)\right|_{y=y_{\mathrm{c}}}$ is negative, that is in our case $y_{\mathrm{c}}>0,\left(C_{+\infty}-C_{-\infty}\right)>0$ resulting in over-reflection and over-transmission. For $y_{\mathrm{c}}<0,\left(\mathrm{~d}^{2} U / \mathrm{d} y^{2}\right) /\left.(\mathrm{d} U / \mathrm{d} y)\right|_{y=y_{\mathrm{c}}}$ is positive and $\left(C_{+\infty}-C_{-\infty}\right)<0$, implying resonant absorption of wave energy at the $\Omega$-critical level. 


\section{NUMERICAL RESULTS}

For $k \neq 0$, the singular wave equation in Eq. (6) seems too complicated to allow for any analytical solutions. Therefore, Eq. (6) is integrated numerically. Reflection and transmission coefficients are then calculated for several values of the wave parameters.

\section{(a) Numerical solution method}

Equation (6) is solved numerically using a 7-8th-order Runge-Kutta algorithm. The step size is automatically reduced when the desired accuracy is not found. An imaginary component of about $10^{-8}$ for the wave frequency $\omega$ is introduced, which acts as a small linear Rayleigh damping. As a consequence, the singularities are then shifted off the real $y$-axis which enables integration along this axis. The integration is started for large positive values of $y$ with a transmitted wave. Equation (6) is then integrated backwards along the $y$-axis to large negative values of $y$ where the solution is split into an incident and a reflected wave.

The ability of the numerical code to integrate across regions where singularities occur is tested in two ways. First, Eq. (6) with $k=0$ and for a hyperbolic tangent profile (Eq. (5)) is integrated numerically and reflection and transmission coefficients are computed. Analytical expressions for reflection and transmission coefficients are also derived by solving Eq. (6) in terms of hypergeometric functions. The numerical and analytical results are compared and the difference is less than $0.005 \%$. Note that for $k=0$, Eq. (6) contains no $\Omega$-type singularity but only an apparent $A$-type singularity. The code integrates through regions where $A=0$, but as expected, no singular behaviour is observed in the solution $\widehat{\phi}$.

Another test for the code is provided by the analytical results of van Duin and Kelder (1982). Their Eq. (2.1) is integrated using the Runge-Kutta code, after which reflection and transmission coefficients are determined. The numerical results are compared with their analytical expressions for the reflection and transmission coefficients (their Eqs. (4.11) and (4.12)). The differences between the numerical and analytical results are of the same order as above.

\section{(b) Choice of parameters}

Realistic values, that are appropriate to the austral winter and early spring polar vortex, are chosen for the parameters $f, N, L$ and $U_{0}$. The value of the Coriolis parameter is calculated at $65^{\circ} \mathrm{S}$ yielding $-1.3 \times 10^{-4} \mathrm{~s}^{-1} .65^{\circ} \mathrm{S}$ corresponds roughly with the average location of the polar-vortex edge derived from European Centre for Medium-Range Weather Forecasts analyses for August 1998. From these same analyses, maximum zonal wind speeds in the Antarctic polar-vortex edge are found to be around $60 \mathrm{~m} \mathrm{~s}^{-1}$, and the width of the polar-vortex edge is estimated to be of the order $5 \times 10^{5} \mathrm{~m}$. The Brunt-Väisälä frequency $N$ in the lower stratosphere is of the order $2 \times 10^{-2} \mathrm{~s}^{-1}$ (Pfenninger et al. 1999; Yoshiki and Sato 2000). The scale height $H$ is taken to be $7 \mathrm{~km}$. The Rossby number (Ro) is defined as Ro $=U_{0} /|f| L$. Hereafter, Ro is set to 1.0 for inertially stable flows and for inertially unstable flows Ro is kept constant at 4.5. The horizontal wavelength, corresponding to $k$, will vary between approximately 100 and $1000 \mathrm{~km}$ and the horizontal wavelength in the $y$-direction, corresponding to $l_{-\infty}$, is taken to be approximately $314 \mathrm{~km}$. The vertical wavelength is taken to be $5 \mathrm{~km}$. An IGW incident upon a background flow will be determined by its wave frequency $\omega$ and its parallel, transverse and vertical wave numbers $k, l$ and $m$, respectively. We assume an IGW incident from $y=-\infty$ having a real transverse wave number $l_{-\infty}>0$, to ensure propagation toward the shear layers, onto the background 


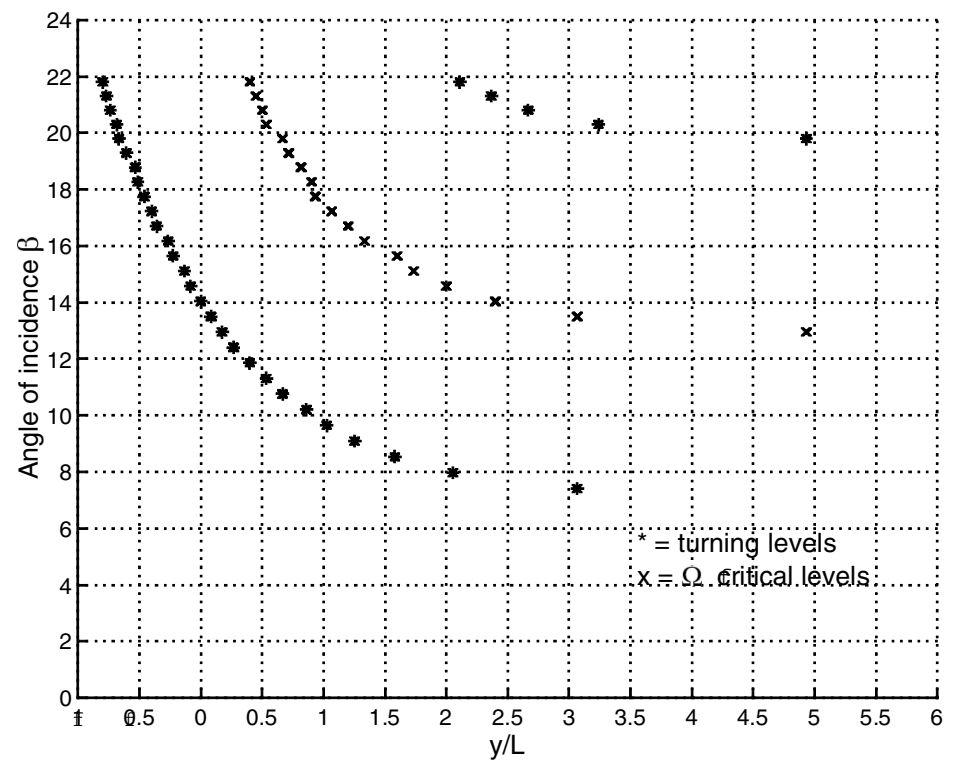

Figure 1. Locations of the turning levels and $\Omega$-critical levels as a function of the angle of incidence $\beta$. The background flow is inertially stable.

flow. The subscript ' $-\infty$ ' in $l_{-\infty}$ indicates the value of $l$ at $y=-\infty$. Then two angles of incidence can be defined for an IGW incident upon a background flow viz.

$$
\alpha=\arctan \left(\frac{m}{l_{-\infty}}\right), \quad \beta=\arctan \left(\frac{k}{l_{-\infty}}\right) .
$$

Using the parameter values above, $\alpha$ is then equal to and taken to be constant at approximately 89 degrees. The angle of incidence $\beta$ will vary. Typical values for the wave frequency $\omega$ are between 1 and 5 times the Coriolis frequency $f$. Overall these values are in good agreement with recent observations made by Guest et al. (2000) for IGWs in the southern-hemisphere lower stratosphere.

\section{(c) Results}

(i) Inertially stable background flow. In Fig. 1 the locations of the turning levels and $\Omega$-critical levels ( $\Omega$-CLs hereafter) are plotted as a function of the angle of incidence $\beta$. From this figure, the following four cases can be distinguished for increasing $\beta$ :

1(a) No turning levels and no $\Omega$-CLs.

2(a) One turning level and no $\Omega$-CLs.

3(a) One turning level followed by one $\Omega-C L$.

4(a) One $\Omega$-CL surrounded by two turning levels.

For $\beta$ larger than 22 degrees, the $\Omega$-CL gradually shifts towards negative values of $y$, but is always surrounded by two turning levels. In fact, close inspection of the behaviour of $Q$ in Eq. (9) shows that a third turning level is present in the flow which nearly coincides with the $\Omega$-CL. However, the distance between this turning level and the $\Omega-C L$ is much smaller than the wavelength. Therefore, this turning level will not have any significant effect on the propagational behaviour of the wave and will be excluded from any further discussion below. In Fig. 2 reflection and transmission 


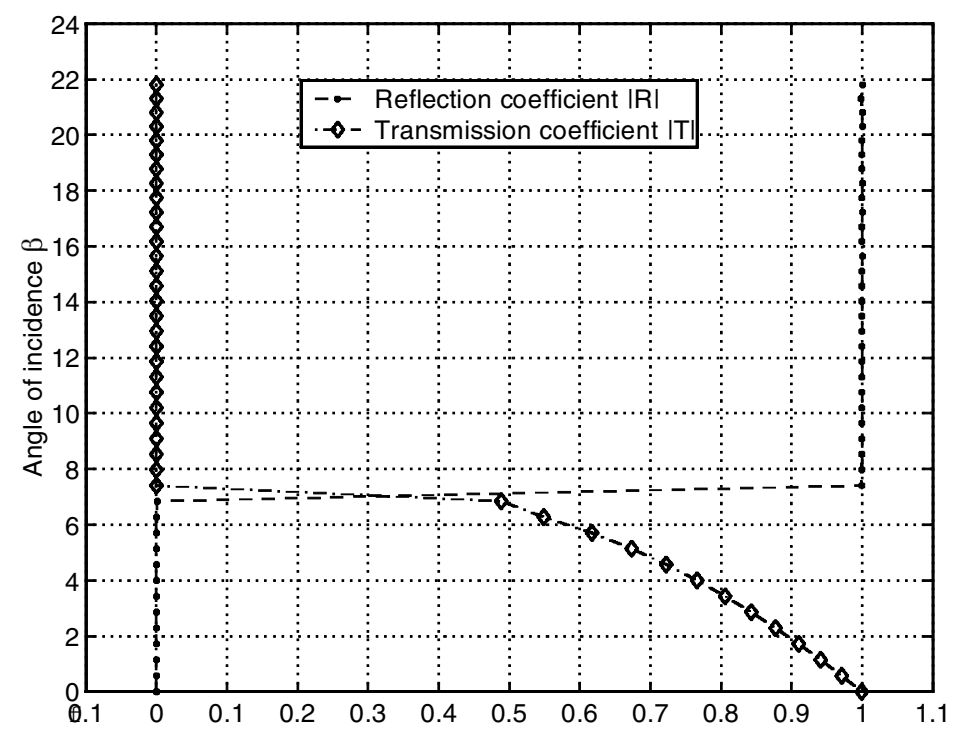

Figure 2. Reflection and transmission coefficients as a function of the angle of incidence $\beta$. The background flow is inertially stable.

coefficients are plotted for the same values of $\beta$ as in Fig. 1. For all values of $\beta$ in Fig. $2,|R|^{2}+(l / A)_{\infty}(A / l)_{-\infty}|T|^{2}=1$. This may appear rather surprising, since one might expect over-reflection, over-transmission or absorption to occur in the presence of an $\Omega$-CL (see appendix). However, for the inertially stable flow it is always found that the distance between the first turning level and the $\Omega$-CL is so large, that the wave has become evanescent $\left(\widehat{\phi}=0^{*}\right)$ by the time it reaches the $\Omega$-CL. Note that $|R|^{2}+(l / A)_{\infty}(A / l)_{-\infty}|T|^{2}=1$ is also found for other values of $\alpha$ and $\beta$.

If there are no turning levels and no $\Omega$-CLs in the flow (case $1(\mathrm{a})$ ), then we have, for the parameters considered, $|R|=0$ always.

For a small range of values of $\beta$, we have one turning level and no $\Omega$-CLs (case 2(a)). In these cases we have $|R|=1$. An example of this is shown in Fig. 3 for the inertially stable flow $\left(\omega_{i}^{2} / f^{2}>0\right.$ for all $\left.y / L\right)$. The angle of incidence $\beta=11$ degrees. The turning level (indicated as 'TL') occurs where $Q=0$. Note that $Q N^{2} / f^{2} L^{2}$ becomes singular where $A=0$ in agreement with Eq. (9). The wave $(\operatorname{Re}(\phi))$ is totally reflected $(|R|=1)$ by the turning level. Since there is only one turning level, the wave becomes evanescent at $y=+\infty$, which explains $|T|=0$.

For $\beta$ larger than approximately 13 degrees, we have a situation in which there is one turning level followed by one $\Omega$-CL in the flow (case 3(a)). In these cases we have $|R|=1$. An example of such a situation is shown in Fig. 4. The parameter values are the same as in Fig. 3, except that $\beta=17$ degrees. The flow is inertially stable $\left(\omega_{i}^{2} / f^{2}>0\right.$ for all $y / L)$. As the wave propagates towards the background flow, it first encounters the turning level $(Q=0)$ and then the $\Omega$-CL $(\Omega=0)$. Similarly to Fig. 3, $Q N^{2} / f^{2} L^{2}$ becomes singular where $A=0$, but also where $\Omega=0$. Note that the wave is again completely reflected. At the turning level the wave becomes evanescent and by the time it reaches the $\Omega-C L$, its amplitude is insignificantly small $(\operatorname{Re}(\phi)=0)$. As mentioned above, the distance between the turning level and the $\Omega-C L$ is so large, that the $\Omega-C L$ has no significant effect on the propagation properties of the wave.

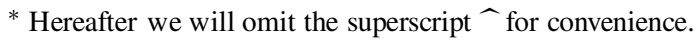




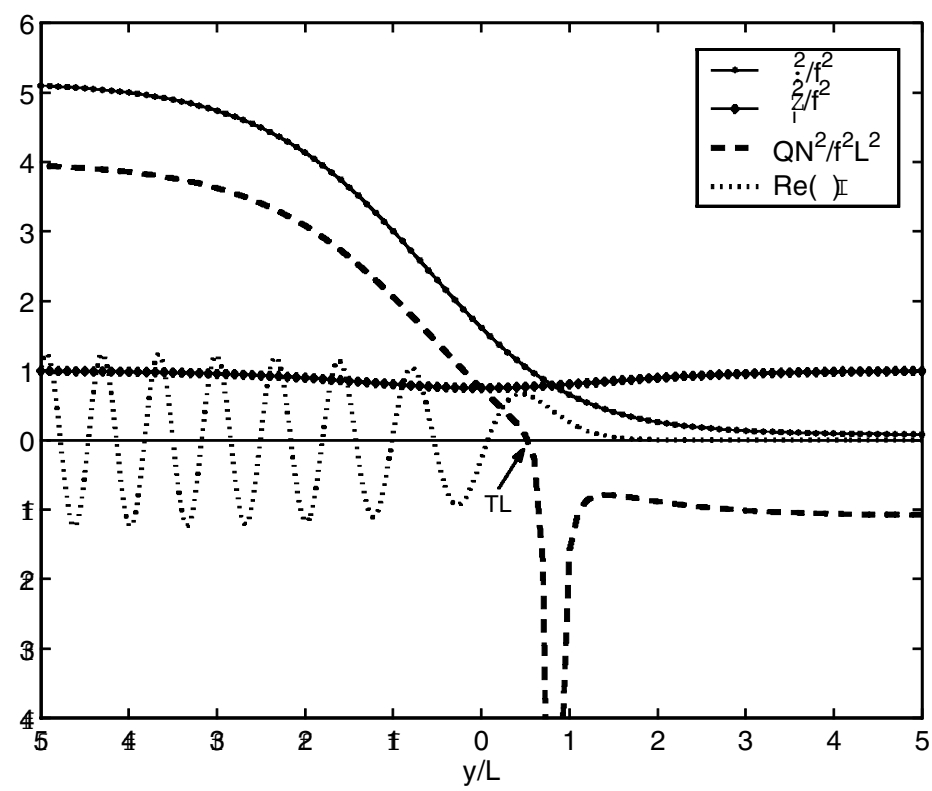

Figure 3. Situation with one turning level and no $\Omega$-critical levels $\left(\Omega^{2} / f^{2}>0\right)$ for a stable background flow $\left(\omega_{i}^{2} / f^{2}>0\right)$. The wave field is represented by $\operatorname{Re}(\phi)$. The turning level ('TL') occurs at the location where $Q=0$. $Q N^{2} / f^{2} L^{2}$ becomes singular where $A=0$, which follows from Eq. (9). The angle $\beta$ is equal to 11 degrees, the reflection coefficient $|R|=1$, and the transmission coefficient $|T|=0$. See text for further information.

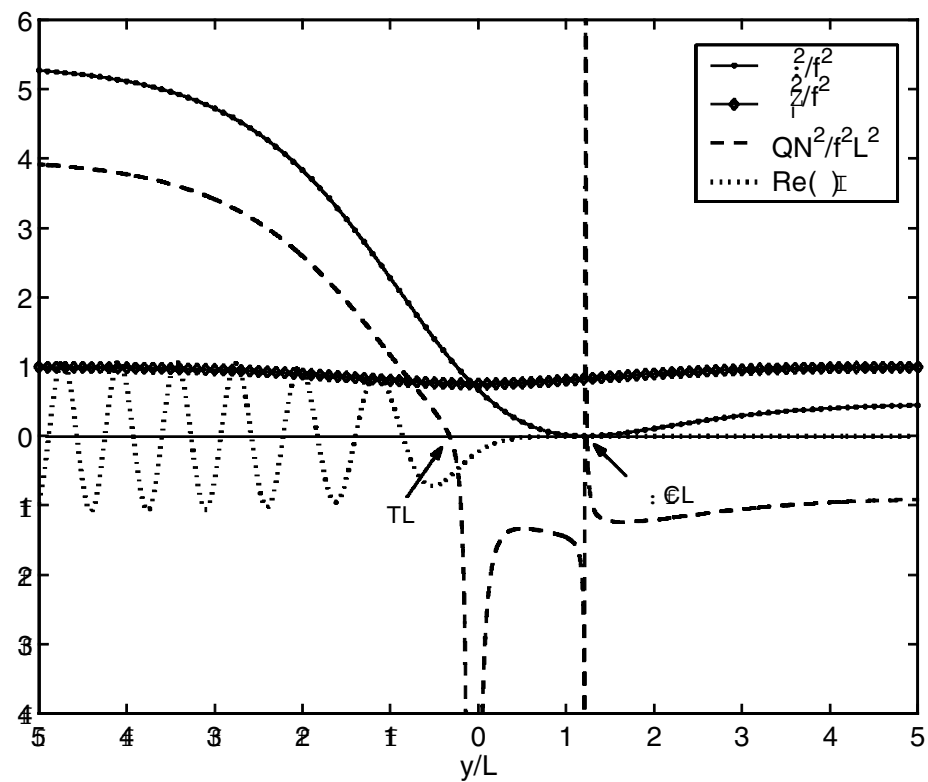

Figure 4. Similar to Fig. 1, but now there is one turning level and one $\Omega$-critical level (' $\Omega$-CL') $(\Omega=0)$ present in the flow. Note that $Q N^{2} / f^{2} L^{2}$ now becomes singular at locations $\Omega=0$ and $A=0$. The angle of incidence $\beta=17$ degrees, the reflection coefficient $|R|=1$, and the transmission coefficient $|T|=0$. See text for further information. 


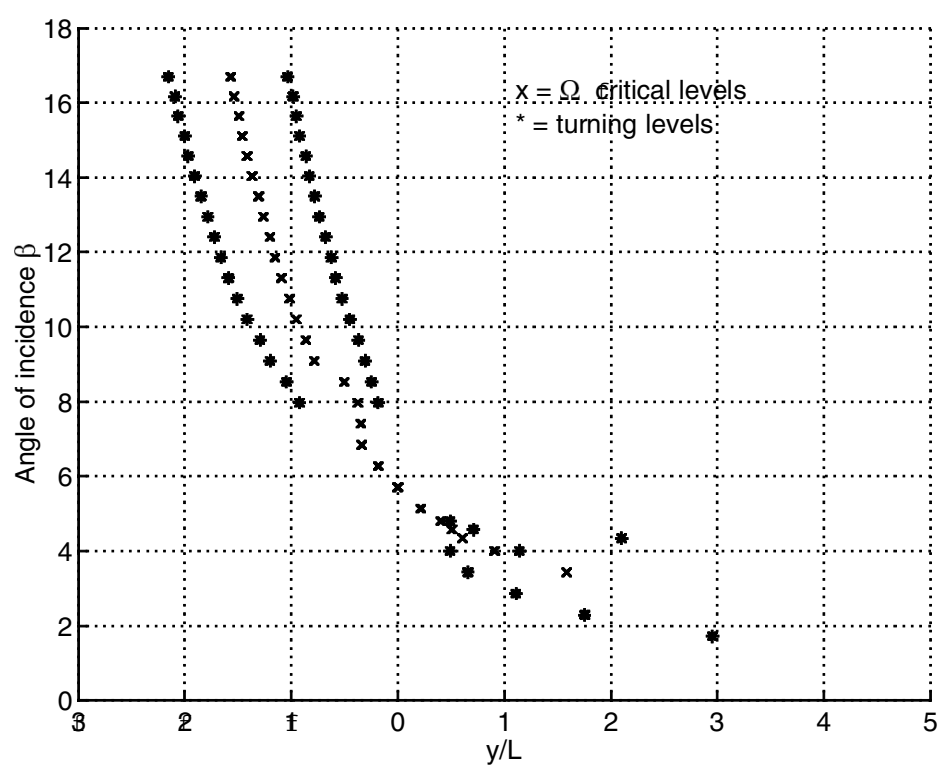

Figure 5. Locations of the turning levels and $\Omega$-critical levels as a function of the angle of incidence $\beta$. The background flow is inertially unstable.

A situation in which the $\Omega$-CL is surrounded by two turning levels (case 4(a)) is reached for $\beta$ larger than approximately 20 degrees. As can be seen from Fig. 2, we have then $|R|=1$ and $|T|=0$ always. The wave is, similarly to the case with one turning level and one $\Omega$-CL, totally reflected. The distance between the (first) turning level and the $\Omega$-CL is in this case also so large that the wave has become evanescent when it reaches the $\Omega$-CL. As a consequence, the wave behaviour is not significantly influenced by the presence of the $\Omega-C L$.

(ii) Inertially unstable background flow. The background flow becomes inertially unstable, around the inflection point $y=0$, for Rossby numbers larger than 4.0. Now reflection and transmission coefficients can become larger or smaller than 1, indicating exchange of momentum and energy between the wave and the background flow. In Fig. 5 the locations of the turning levels and the $\Omega$-CLs are plotted as a function of the angle of incidence $\beta$. From this figure, six different cases can be identified:

1(b) No turning levels and no $\Omega$-CL.

2(b) One turning level and no $\Omega$-CL.

3(b) One turning level followed by one $\Omega$-CL.

4(b) One $\Omega$-CL followed by one turning level.

5 (b) No turning levels and one $\Omega$-CL.

6(b) One $\Omega$-CL surrounded by two turning levels.

Similarly to the inertially stable case, turning levels are also present very close to the location of the $\Omega$-CLs. For the same reasons as in the inertially stable case, we will not discuss these turning levels further. Reflection and transmission coefficients are plotted in Fig. 6 for the same values of $\beta$ as in Fig. 5. Note from Fig. 5 and Fig. 6 that over-reflection and over-transmission occur in the case when the location of the $\Omega$-CL $y_{\mathrm{c}} / L>0$, and that absorption occurs in the case when the location of the $\Omega$-CL $y_{\mathrm{c}} / L<0$. In the appendix it is shown that the location of $y_{\mathrm{c}} / L$, is related 


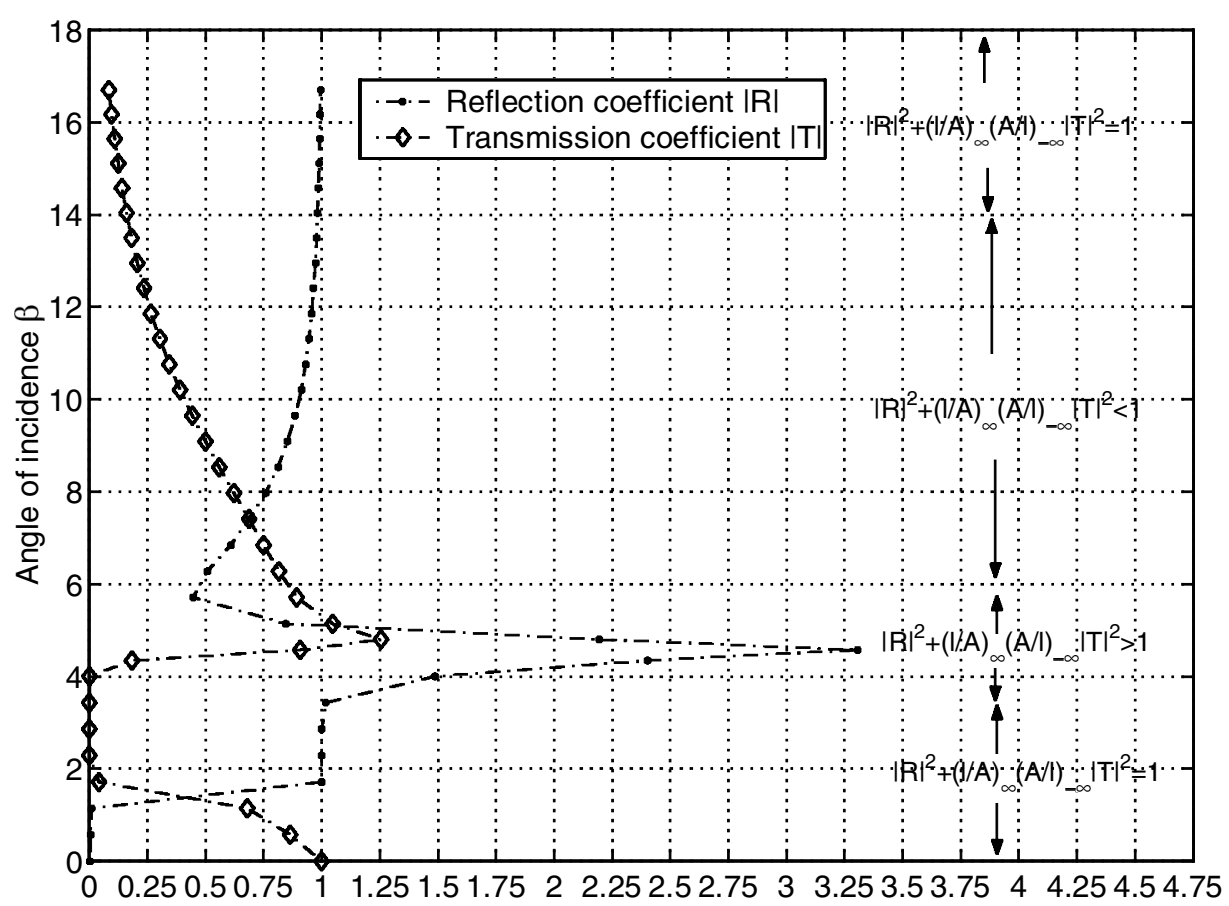

Figure 6. Reflection and transmission coefficients as a function of the angle of incidence $\beta$. The background flow is inertially unstable. The range of values of $\beta$ where over-reflection and over-transmission $\left(|R|^{2}+\right.$ $\left.(l / A)_{\infty}(A / l)_{-\infty}|T|^{2}>1\right)$ and absorption $\left(|R|^{2}+(l / A)_{\infty}(A / l)_{-\infty}|T|^{2}<1\right)$ occurs is also indicated. See text for further information.

to the sign of $-\left(\mathrm{d}^{2} U / \mathrm{d} y^{2}\right) /(\mathrm{d} U / \mathrm{d} y)$ at the location of the critical level. The sign of $-\left(\mathrm{d}^{2} U / \mathrm{d} y^{2}\right) /(\mathrm{d} U / \mathrm{d} y)$ at the critical level determines, in its turn, the sign in the jump of the momentum flux. For example if $y_{\mathrm{c}} / L>0$, then $-\left(\mathrm{d}^{2} U / \mathrm{d} y^{2}\right) /(\mathrm{d} U / \mathrm{d} y)>0$ yielding a positive jump in the momentum flux. From Eq. (A.8) and Eq. (21) it can then be seen that a positive jump in the momentum flux corresponds to over-reflection and over-transmission. A similar reasoning can be followed to explain the occurrence of absorption in case $y_{\mathrm{c}} / L<0$.

Based on the different cases above it can be seen that in case $1(\mathrm{~b}),|R|$ is nearly zero and $|T|$ is approximately between 0.6 and 1.0 and $|R|^{2}+(l / A)_{\infty}(A / l)_{-\infty}|T|^{2}=1$.

In cases 2(b) and 3(b) we have $|R|=1$ and $|T|=0$ always, as in the inertially stable case.

Over-reflection $(|R|>1)$ and over-transmission $(|T|>1)$ occurs for $\beta$ between approximately 4 and 5 degrees. Note from Fig. 6 that over-reflection and over-transmission is most pronounced in the case when there is only one $\Omega$-CL followed by a turning level (case 4(b)) or in the case when there is one $\Omega$-CL only (case 5(b)). An example in which spectacular over-reflection occurs is shown in Fig. 7, with $|R|=3.304,|T|=0.906$ and $|R|^{2}+(l / A)_{\infty}(A / l)_{-\infty}|T|^{2}=12.689$. Note that the $\Omega$-CL is located in the region where the flow is inertially unstable $\left(\omega_{i}^{2} / f^{2}<1\right.$ for certain values of $\left.y / L\right)$.

For $\beta$ between approximately 5 and 14 degrees absorption with $|R|<1$ and $|T|<1$ is dominant (cases 5(b) and 6(b)). An example of this is shown in Fig. 8 for $\beta \approx$ 5.7 degrees with $|R|=0.448,|T|=0.891$ and $|R|^{2}+(l / A)_{\infty}(A / l)_{-\infty}|T|^{2}=0.991$. There is only one $\Omega$-CL located in the flow and no turning levels. Similarly to Fig. 7, 


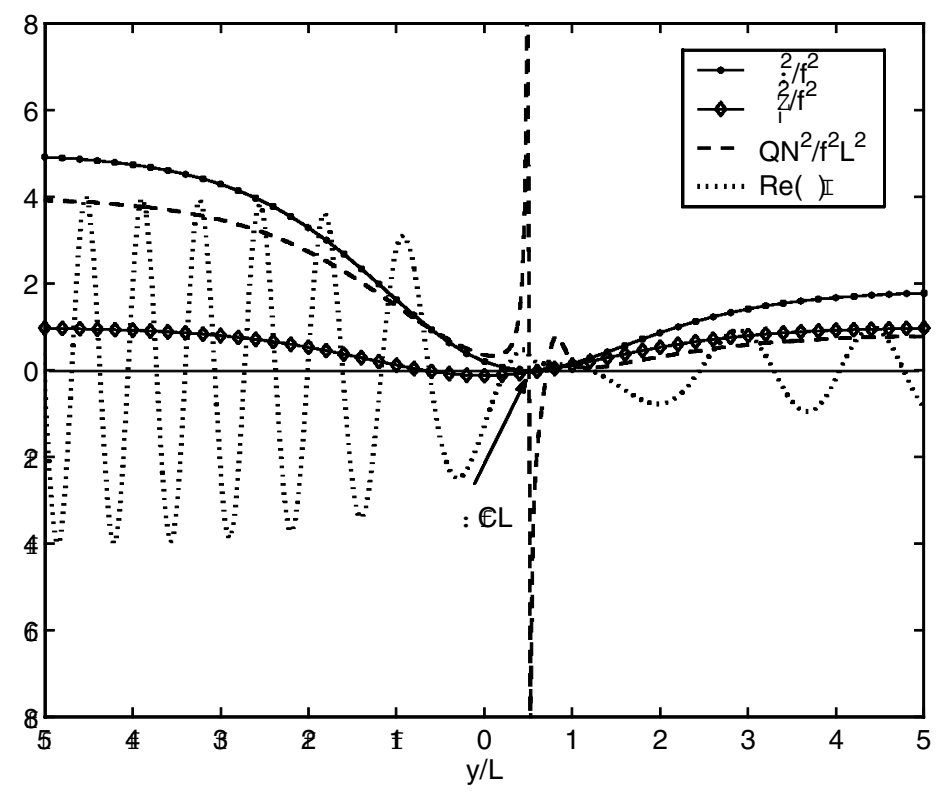

Figure 7. An example of over-reflection with $|R|=3.304,|T|=0.906$ and $|R|^{2}+(l / A)_{\infty}(A / l)_{-\infty}|T|^{2}=$ 12.689. The plotted quantities are equal to those in Fig. 1 and Fig. 2. The flow is inertially unstable $\left(\omega_{i}^{2}<0\right)$ between approximately $-1<y<1$. Singular behaviour in $Q N^{2} / f^{2} L^{2}$ occurs at locations where $\Omega=0$. The angle of incidence $\beta \approx 4.5$ degrees. See text for further information.

the $\Omega$-CL is located in the region where the flow is inertially unstable $\left(\omega_{i}^{2} / f^{2}<0\right.$ for certain values of $y / L$ ) and $Q N^{2} / f^{2} L^{2}$ becomes singular at the $\Omega$-CL.

Another example of absorption is shown in Fig. 9 for $\beta \approx 10$ degrees. Here $|R|=0.912,|T|=0.379$ so $|R|^{2}+(l / A)_{\infty}(A / l)_{-\infty}|T|^{2}=0.886$. Now we have one $\Omega$-CL that is surrounded by two turning levels. The quantity $Q N^{2} / f^{2} L^{2}$ shows singular behaviour at the $\Omega-\mathrm{CL}$ and where $A=0$ (see Eq. (9)). It is interesting to note that the $\Omega$-CL is now located just outside the region where the flow is inertially unstable $\left(\omega_{i}^{2} / f^{2}<0\right)$.

For values of $\beta$ larger than approximately 14 degrees, the distance between the first turning level and the $\Omega$-CL increases, so we finally recover that $|R| \simeq 1$ and $|T| \simeq 0$. This is in agreement with the results for the stable background profile. As soon as the distance between the first turning level and the $\Omega$-CL becomes too large, the wave has become completely evanescent when it reaches the $\Omega$-CL. The $\Omega$-CL will then have an insignificant effect on the wave behaviour.

The fact that the $\Omega$-CL in Fig. 7 and Fig. 8 is located in the region where the background flow is inertially unstable is not directly related to the occurrence of overreflection, over-transmission and absorption. This is confirmed by Fig. 9 where an example of absorption is shown, but where the $\Omega$-CL is located outside the region where the background flow is inertially unstable. The location of the $\Omega$-CLs and turning levels is determined by $\beta$ and by $Q$ in Eq. (9). Nevertheless, from Eq. (9) it can be seen that the sign of $\omega_{i}^{2} / f^{2}$ does, to some degree, influence the behaviour of $Q$. However, the actual occurrence of over-reflection, over-transmission and absorption is determined by the location of the $\Omega$-CL in the flow, which determines the sign of the jump in the momentum flux (appendix), and by the distance between the first-occurring turning level and the $\Omega-C L$. 


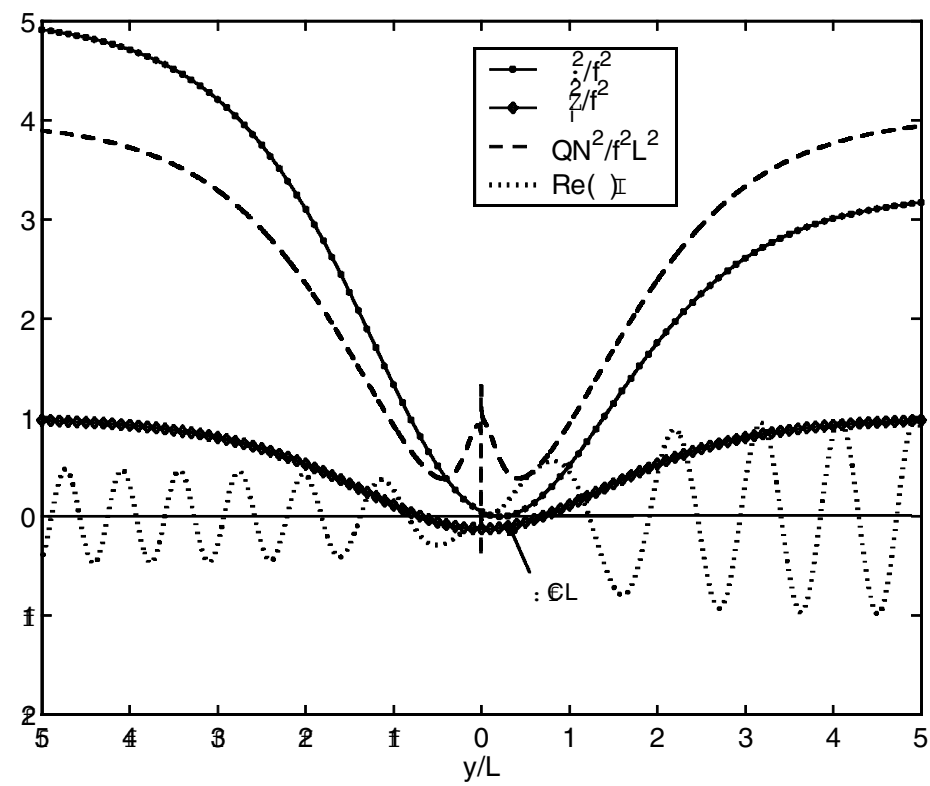

Figure 8. An example of an inertially unstable background flow, with one $\Omega$-critical level and no turning levels. The plotted quantities are equal to those in Fig. 1 and Fig. 2. The reflection coefficient $|R|$ is equal to 0.448 and $|T|$ is equal to 0.891 and $|R|^{2}+(l / A)_{\infty}(A / l)_{-\infty}|T|^{2}=0.991$. Singular behaviour in $Q N^{2} / f^{2} L^{2}$ occurs at the location where $\Omega=0$. The angle of incidence $\beta \approx 5.7$ degrees. See text for further information.

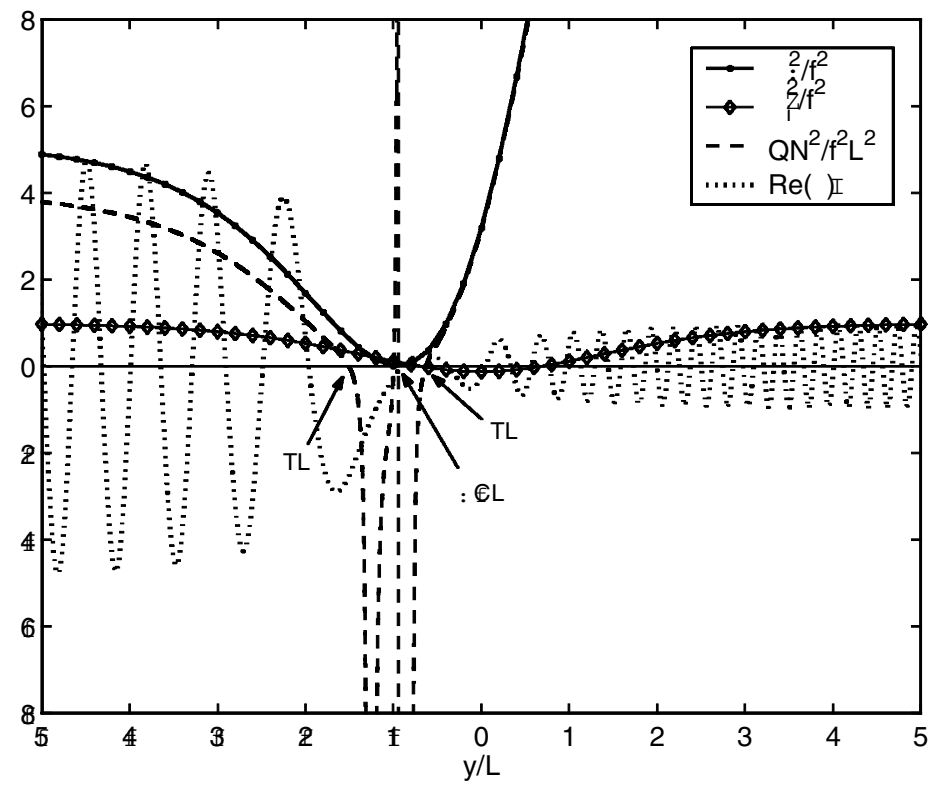

Figure 9. An example of absorption with $|R|=0.912,|T|=0.379$ and $|R|^{2}+(l / A)_{\infty}(A / l)_{-\infty}|T|^{2}=0.886$. The plotted quantities are equal to those in Fig. 1 and Fig. 2. Singular behaviour in $Q N^{2} / f^{2} L^{2}$ occurs at locations where $\Omega=0$ and $A=0$. The angle of incidence $\beta \approx 10$ degrees. See text for further information. 
Other experiments with different values for $\alpha$ and Ro (>4.0) do not significantly alter the results presented above.

\section{Conclusions}

In this study a linear model is presented describing the propagation of IGWs on an $f$-plane in a vertically homogeneous background flow with shear in the latitudinal direction. The resulting wave equation contains two types of singularities. One corresponds to the occurrence of a critical level in the flow when the intrinsic wave frequency $\Omega$ is equal to zero, here referred to as the $\Omega$-CL. This singularity is similar to the classical Booker and Bretherton type of singularity for horizontally homogeneous background flows with vertical shear. The other type of singularity is an apparent singularity and has been discussed in the literature in different contexts.

Numerical integration of the singular wave equation enables the calculation of reflection and transmission coefficients for a wide range of parameter values and for two angles of incidence $\alpha=m / l_{-\infty}$ and $\beta=k / l_{-\infty}$.

The main results can be summarized as follows:

- The following distinction can be made for an inertially stable background flow:

- $\quad|R|=0$ and $|T|<1$ in cases when there are no turning levels and no $\Omega$-CLs.

- $\quad|R|=1$ and $|T|=0$ when the wave encounters one turning level only.

- $\quad|R|=1$ and $|T|=0$ when the wave first encounters a turning level followed by an $\Omega$-CL.

- $\quad$ In all cases above $|R|^{2}+(l / A)_{\infty}(A / l)_{-\infty}|T|^{2}=1$.

Also, in all cases considered for the stable background flow where $|R|=1$ and $|T|=0$, the distance between the (first) turning level and the $\Omega$-CL played an important role. When the wave passes the turning level it becomes evanescent and its amplitude has become insignificantly small when reaching the $\Omega$-CL. The wave reflects completely at the turning level and the $\Omega$-CL has no significant effect on the wave propagation. This explains the robust result $|R|=1$ and $|T|=0$.

- For inertially unstable background flows the following distinction can be made:

- $\quad|R|=0$ and $|T|<1$ when there are no turning levels and no $\Omega$-CLs.

- $\quad|R|=1$ and $|T|=0$ when the wave encounters a turning level only.

- $\quad$ In the two cases above we have $|R|^{2}+(l / A)_{\infty}(A / l)_{-\infty}|T|^{2}=1$.

- Over-reflection $(|R|>1)$ and over-transmission $(|T|>1)$ occurs mostly in the case when there is only one $\Omega$-CL in the flow. In a few cases this $\Omega$-CL is followed by a turning level.

- Absorption $(|R|,|T|<1)$ occurs mostly in the case when one $\Omega$-CL is surrounded by two turning levels. As the distance between the first turning level and the $\Omega$-CL increases for increasing $\beta$, finally $|R| \simeq 1$ and $|T| \simeq 0$.

- The sign of $-\left(\mathrm{d}^{2} U / \mathrm{d} y^{2}\right) /(\mathrm{d} U / \mathrm{d} y)$ at the location of the $\Omega$-CL determines the sign of the jump in the momentum flux, which in its turn determines whether over-reflection/over-transmission or absorption occurs.

An important finding in this study, is the fact that in inertially stable flows the wave will always totally reflect at the turning level and never reach the critical level. In inertially unstable flows the turning level can disappear and the wave can reach the critical level. At the critical level over-reflection, over-transmission and absorption can then occur. 
In this study we have not accounted for the effects of wave saturation and eventually wave breaking, processes that may significantly contribute to the exchange across the vortex edge. Nevertheless, our results show a wide range of behaviour that may occur when IGWs propagate in shear flows like the polar vortex edge.

\section{ACKNOWLEDGEMENTS}

M. C. Öllers wishes to thank the Netherlands Science Foundation (NWO) for their support under project STRATEOLE-NL (the study of stratospheric dynamics and ozone chemistry in the southern hemisphere, the Netherlands) and Dr F. Vial (Laboratoire de Météorologie Dynamique du CNRS, Paris) for the participation in the international STRATEOLE project. The authors would also like to thank two anonymous referees and T. J. Dunkerton for their useful comments during the review stage, which greatly improved the manuscript.

\section{APPENDIX}

Jump in momentum flux across the $\Omega$-critical level

In section 2(c) it is shown that whenever $\Omega$ becomes zero somewhere between $y=-\infty$ and $y=+\infty$, the momentum flux in Eq. (12) can jump in passing critical levels. These jumps are related to logarithmic singularities that are analysed below.

At the critical level $y=y_{\mathrm{c}}$ where $\Omega(y)=0$, a Taylor expansion of $\Omega(y)$ around $\tilde{y}=y-y_{\mathrm{c}}$ can be made i.e.

$$
\Omega(y)=\Omega_{1} \tilde{y}+\Omega_{2} \widetilde{y}^{2}+\cdots
$$

where $\Omega_{1}=\Omega_{\widetilde{y}}(0)$ and $\Omega_{2}=1 / 2 \Omega_{\widetilde{y y}}(0)$.

In a similar way the Taylor expansion for $A(y)$ around $\tilde{y}=0$ is given by

$$
A(y)=A_{1}(\tilde{y})+A_{2}(\widetilde{y})^{2}+\cdots
$$

where $A_{1}=A_{\widetilde{y}}(0)$ and $A_{2}=1 / 2 A_{\widetilde{y y}}(0)$. We note that the expansion coefficients $\Omega_{1}$, $\Omega_{2}, \ldots, A_{1}, A_{2}, \ldots$ are all related to the Taylor expansion coefficients of the background flow $U(y)$.

Application of the method of Frobenius to Eq. (6) and use of Eqs. (A.1) and (A.2) yields Frobenius exponents 0 and 1 . The first solution reads

$$
\widehat{\phi}_{\Omega_{1}}(\widetilde{y})=\tilde{y} \sum_{n=0}^{\infty} c_{n} \widetilde{y}^{n}, \quad c_{0} \neq 0, \quad \text { where } \quad \tilde{y}=y-y_{\mathrm{c}},
$$

and a second linearly independent solution is given by

$$
\widehat{\phi}_{\Omega_{2}}(\widetilde{y})=\widehat{\phi}_{\Omega_{1}}(\widetilde{y})\left(B_{\Omega_{1}} \ln \tilde{y}+B_{\Omega_{2}} \tilde{y}^{-1}\right)+\widehat{\phi}_{\Omega_{1}}(\widetilde{y}) \sum_{n=2}^{\infty} \frac{\mathrm{d}_{n}}{n-1} \widetilde{y}^{n-1},
$$

where $B_{\Omega_{1}}$ and $B_{\Omega_{2}}$ are constants defined as

$$
B_{\Omega_{1}}=\frac{A_{1}}{a_{0}^{2}}-\frac{2 a_{1}}{a_{0}^{3}} A_{0}, \quad B_{\Omega_{2}}=-\frac{A_{0}}{a_{0}^{2}} .
$$

The solution can again be written as a linear combination of Eqs. (A.3) and (A.4), i.e.

$$
\widehat{\phi}_{\Omega}(\tilde{y})=F_{\Omega} \widehat{\phi}_{\Omega_{1}}(\widetilde{y})+G_{\Omega} \widehat{\phi}_{\Omega_{2}}(\widetilde{y}) .
$$


Recalling Eq. (11), the jump across $\Omega$-critical levels can now be determined from

$$
\begin{aligned}
\Delta \operatorname{Re}\left(\frac{\mathrm{i} \hat{\phi}^{*}}{A} \frac{\mathrm{d} \widehat{\phi}}{\mathrm{d} y}\right)=\Delta & \operatorname{Re}\left\{\frac { \mathrm { i } } { A } \left(|F|_{\Omega_{2}}^{2} \widehat{\phi}_{\Omega_{1}}^{*} \frac{\mathrm{d} \widehat{\phi}_{\Omega_{1}}}{\mathrm{~d} y}+|G|_{\Omega}^{2} \widehat{\phi}_{\Omega_{2}}^{*} \frac{\mathrm{d} \widehat{\phi}_{\Omega_{2}}}{\mathrm{~d} y}\right.\right. \\
& \left.\left.+F_{\Omega}^{*} G_{\Omega} \widehat{\phi}_{\Omega_{1}}^{*} \frac{\mathrm{d} \widehat{\phi}_{\Omega_{2}}}{\mathrm{~d} y}+F_{\Omega} G_{\Omega}^{*} \widehat{\phi}_{\Omega_{2}}^{*} \frac{\mathrm{d} \widehat{\phi}_{\Omega_{1}}}{\mathrm{~d} y}\right)\right\} .
\end{aligned}
$$

All terms in Eq. (A.7) give zero contributions at $\tilde{y}=0$, except the term proportional to $|G|_{\Omega}^{2}$. After some algebra Eq. (A.7) takes the form

$$
C_{+\infty}-C_{-\infty} \equiv \Delta \operatorname{Re}\left(\frac{\mathrm{i} \widehat{\phi}^{*}}{A} \frac{\mathrm{d} \widehat{\phi}}{\mathrm{d} y}\right)=|G|_{\Omega}^{2} \Delta \operatorname{Re}\left(\mathrm{i} J_{\Omega} \ln \tilde{y}\right),
$$

where $J_{\Omega}$ is given by

$$
J_{\Omega}=\frac{2 a_{1}\left|A_{0}\right|^{2}-a_{0} A_{1} A_{0}^{*}}{a_{0} A_{0}\left|a_{0}\right|^{2}} .
$$

Evaluating $A_{0}, A_{1}, a_{0}$ and $a_{1}$ in terms of the Taylor expansion coefficients of the background flow $U(y)$, reveals that the sign of $J_{\Omega}$ is determined by the sign of $-\left(\mathrm{d}^{2} U / \mathrm{d} y^{2}\right) /(\mathrm{d} U / \mathrm{d} y)$ at the location of the critical level $y=y_{\mathrm{c}}$.

From Eq. (12) the momentum flux can be written as

$$
\Delta \tau=\rho_{0} k|G|_{\Omega}^{2}\left\langle\Delta \operatorname{Re}\left(\mathrm{i} J_{\Omega} \ln \tilde{y}\right)\right\rangle,
$$

for real $k$. Obviously the sign of the jump is determined by the sign of $J_{\Omega}$. For the hyperbolic tangent profile this means that we have a positive jump in the momentum flux for $y_{\mathrm{c}}>0$ and a negative jump for $y_{\mathrm{c}}<0$.

Acheson, D. J.

Andrews, D. G., Holton, J. R. and Leovy, C. B.

Basovich, A. Ya. and

Tsimring, L. Sh.

Booker, J. R. and Bretherton, F. P. 1967

Bowman, K. P.

Boyd, J. P.

Chen, $\mathrm{P}$.

Dunkerton, T. J.

Flattery, T. W.

Guest, F. M., Reeder, M. J., Marks, C. J. and Karoly, D. J. Ivanov, Y. A. and Morozov, Y. G.
1976

1987

1984

1993

1976

1978

1994

1984

1990

1967

2000

1974

\section{REFERENCES}

On over-reflection. J. Fluid Mech., 77, 433-472

Middle atmosphere dynamics. Academic Press, San Diego

Internal waves in a horizontally inhomogeneous flow. J. Fluid Mech., 142, 233-249

The critical layer for internal gravity waves in a shear flow. J. Fluid Mech., 27, 513-539

Barotropic simulation of large-scale mixing in the Antarctic polar vortex. J. Atmos. Sci., 50, 2901-2914

'Planetary waves and the semiannual wind oscillation in the tropical lower stratosphere'. PhD thesis, Harvard University

The effects of latitudinal shear on equatorial waves. Part 1: Theory and methods. J. Atmos. Sci., 35, 2236-2258

Permeability of the Antarctic vortex edge. J. Geophys. Res., 99, 20563-20571

Inertia-gravity waves in the stratosphere. J. Atmos. Sci., 41, 33963404

Eigenfrequencies and horizontal structure of divergent barotropic instability originating in tropical latitudes. J. Atmos. Sci., 47, 1288-1301

'Hough functions'. Tech. Rep. No. 21, Dept. of Geophysical Sciences, University of Chicago

Inertia-gravity waves observed in the lower stratosphere over Macquarie Island. J. Atmos. Sci., 57, 737-752

Deformation of internal gravity waves by a stream with horizontal velocity shear. Oceanology, 14, 457-461 
Jones, W. L.

1967 Propagation of internal gravity waves in fluids with shear flow and rotation. J. Fluid Mech., 30, 439-448

1968 Reflection and stability of waves in stably stratified fluids with shear flow: A numerical study. J. Fluid Mech., 34, 609-624

Juckes, M. N. and McIntyre, M. E. 1987

Kitchen, E. H. and McIntyre, M. E. 1980

Longuet-Higgins, M. S.

McIntyre, M. E.

Olbers, D.

Öllers, M. C., van Velthoven, P. F. J., Kelder, H. M. and Kamp, L. P. J.

Pfenninger, M., Liu, A. Z., Papen, G. C. and Gardner, Ch. S.

Pierce, R. B., Fairlie, T. D., Grose, W. L., Swinbank, R. and O'Neill, A.

Randel, W.

Schoeberl, M. R. and Hartmann, D. L.

Staquet, C. and Huerre, G.

Van Duin, C. A. and Kelder, H.

Yamanaka, M. D. and Tanaka, H.

Yoshiki, M. and Sato, K.
A high-resolution one-layer model of breaking planetary waves in the stratosphere. Nature, 328, 590-596

On whether inertio-gravity waves are absorbed or reflected when their intrinsic frequency is Doppler-shifted towards $f$. J. Meteorol. Soc. Jpn, 58, 118-125

1968 The eigenfunctions of Laplace's tidal equation over a sphere. Phil. Trans. R. Soc. London A, 262, 511-607

1989 On the Antarctic ozone hole. J. Atmos. Terr. Phys., 51, 29-43

1995 The stratospheric polar vortex and sub-vortex: Fluid dynamics and midlatitude ozone loss. Philos. Trans. R. Soc. London A, 352, 227-240

The propagation of internal waves in a geostrophic current. J. Phys. Oceanogr., 11, 1224-1233

2002 A study of the leakage of the Antarctic polar vortex in late austral winter and spring using isentropic and 3D-trajectories. J. Geophys. Res., doi: 107, 10.1029/2001JD001363

1999 Gravity wave characteristics in the lower atmosphere at the south pole. J. Geophys. Res., 104, 5963-5984

1994 Mixing processes within the polar night jet. J. Atmos. Sci., 51, 2957-2972

1993 Ideal flow on an Antarctic vortex. Nature, 364, 105-106

1991 The dynamics of the stratospheric polar vortex and its relation to springtime ozone depletion. Science, 251, 46-52 waves. Phys. Fluids, 14, 1993-2006

1982 Reflection properties of internal gravity waves incident upon a hyperbolic tangent shear layer. J. Fluid Mech., 120, 505-521

1984 Propagation and breakdown of internal inertio-gravity waves near critical levels in the middle atmosphere. J. Meteorol. Soc. Jpn, 62, 1-17

2000 A statistical study of gravity waves in the polar regions based on operational radiosonde data. J. Geophys. Res., 105, 1799518011
2001 Transport across a barotropic shear flow by breaking gravity 